\title{
SARS-CoV-2 infection in cats and dogs in infected mink farms
}

\author{
Anna E. van Aart ${ }^{1}$ | Francisca C. Velkers ${ }^{2}$ (ㅇ) | Egil A.J. Fischer ${ }^{2}$ () | Els M. Broens ${ }^{3}$ \\ Herman Egberink $^{4}$ | Shan Zhao ${ }^{4}$ | Marc Engelsma ${ }^{5}$ | Renate W. Hakze-van der \\ Honing $^{5}$ | Frank Harders ${ }^{5}$ | Myrna M.T. de Rooij ${ }^{1}$ | Carien Radstake ${ }^{6}$ \\ Paola A. Meijer $^{2}$ | Bas B.Oude Munnink ${ }^{7}$ | Jan de Rond ${ }^{8}$ | Reina S.Sikkema \\ Arco N. van der Spek ${ }^{9}$ | Marcel Spierenburg ${ }^{9}$ | Wendy J. Wolters ${ }^{2}$ \\ Robert-Jan Molenaar $^{8} \quad$ | Marion P.G. Koopmans ${ }^{7} \quad$ Wim H.M. van der Poel ${ }^{5}$ \\ Arjan Stegeman $^{2} \quad$ | Lidwien A.M. Smit ${ }^{1}$ (i)
}

${ }^{1}$ Institute for Risk Assessment Sciences (IRAS), Utrecht University, Utrecht, The Netherlands

${ }^{2}$ Faculty of Veterinary Medicine, Department of Farm Animal Health, Utrecht University,

Utrecht, The Netherlands

${ }^{3}$ Department of Biomolecular Health

Sciences, Infectious Diseases and Immunology,

Clinical Infectiology Division, Utrecht

University, Utrecht, The Netherlands

${ }^{4}$ Department of Biomolecular Health

Sciences, Infectious Diseases and Immunology,

Virology Division, Utrecht University, Utrecht,

The Netherlands

${ }^{5}$ Wageningen Bioveterinary Research, Lelystad, The Netherlands

${ }^{6}$ Stichting Zwerfkatten Nederland (Stray Cat Foundation Netherlands), Nieuw Beijerland, The Netherlands

${ }^{7}$ Department of Viroscience, Erasmus MC, Rotterdam, The Netherlands

${ }^{8}$ GD Animal Health, Deventer, The Netherlands

${ }^{9}$ Netherlands Food and Consumer Product Safety Authority (NVWA), Utrecht, The Netherlands

\section{Correspondence}

Lidwien A.M. Smit, Institute for Risk Assessment Sciences (IRAS), Utrecht University,

Utrecht, The Netherlands.

Email: L.A.Smit@uu.nl

Funding information

Netherlands Ministry of Agriculture, Nature and Foods

\begin{abstract}
Animals like mink, cats and dogs are susceptible to SARS-CoV-2 infection. In the Netherlands, 69 out of 127 mink farms were infected with SARS-CoV-2 between April and November 2020 and all mink on infected farms were culled after SARS-CoV-2 infection to prevent further spread of the virus. On some farms, (feral) cats and dogs were present. This study provides insight into the prevalence of SARS-CoV-2-positive cats and dogs in 10 infected mink farms and their possible role in transmission of the virus. Throat and rectal swabs of 101 cats ( 12 domestic and 89 feral cats) and 13 dogs of 10 farms were tested for SARS-CoV-2 using PCR. Serological assays were performed on serum samples from 62 adult cats and all 13 dogs. Whole Genome Sequencing was performed on one cat sample. Cat-to-mink transmission parameters were estimated using data from all 10 farms. This study shows evidence of SARS-CoV-2 infection in 12 feral cats and 2 dogs. Eleven cats (18\%) and two dogs (15\%) tested serologically positive. Three feral cats (3\%) and one dog (8\%) tested PCR-positive. The sequence generated from the cat throat swab clustered with mink sequences from the same farm. The calculated rate of mink-to-cat transmission showed that cats on average had a chance of $12 \%(95 \% \mathrm{Cl} 10 \%-18 \%$ ) of becoming infected by mink, assuming no cat-to-cat transmission. As only feral cats were infected it is most likely that infections in cats were initiated by mink, not by humans. Whether both dogs were infected by mink or humans remains inconclusive. This study presents one of the first reports of interspecies transmission of SARS-CoV-2 that does not involve humans, namely mink-to-cat transmission, which should also be considered as a potential risk for spread of SARS-CoV-2.
\end{abstract}

\section{KEYWORDS}

cats, dogs, epidemiology, mink, one health, SARS-CoV-2

This is an open access article under the terms of the Creative Commons Attribution-NonCommercial-NoDerivs License, which permits use and distribution in any medium, provided the original work is properly cited, the use is non-commercial and no modifications or adaptations are made.

(c) 2021 The Authors. Transboundary and Emerging Diseases published by Wiley-VCH GmbH 


\section{1 | INTRODUCTION}

Animals like mink, ferrets, dogs, cats and other Felids are susceptible to SARS-CoV-2 infection (Oreshkova et al., 2020; Patterson et al., 2020; Shi et al., 2020 ). In all reported cases, domestic cats and dogs were most likely infected by their owners. Experimental studies have indicated that cat-to-cat transmission is possible (Halfmann et al., 2020; Shi et al., 2020), but evidence of cat-to-human or dog-to-human transmission has not been reported yet (Decaro et al., 2021).

SARS-CoV-2 outbreaks on mink farms have been reported in several countries worldwide (Boklund et al., 2021; Fenollar et al., 2021; Oreshkova et al., 2020 ). In April 2020, the first infected mink farms were detected in the Netherlands (Oreshkova et al., 2020). Before annual pelting took place in November and December, 69 of the 127 Dutch mink farms were infected with SARS-CoV-2. As of June 2020 the Dutch government decided to cull all mink on infected farms to stop spread of SARS-CoV-2. Furthermore, mink farming was banned as of January 2021 (Rijksoverheid, 2020). On the first 16 infected mink farms, $68 \%$ of the farm owners and their family members tested positive for SARS-CoV-2 and whole genome sequencing in two employees provided proof that they had been infected by the virus circulating among mink (Oude Munnink et al., 2021).

Some of the infected mink farms had domestic cats and dogs and/or feral cats that could come in close contact with the mink after entering the mink sheds. These cats could roam on and beyond the farm premises and some were allowed inside the farmer's house. SARS-CoV2 infections and virus shedding in cats and dogs on the infected farms might pose a risk for humans or other animals. Therefore, after culling, farm owners were obliged to keep dogs and cats on the farm premises, as much as this was possible.

We aimed to assess the prevalence of SARS-CoV-2-positive (PCRand/or seropositive) cats and dogs on mink farms and potential risk factors for SARS-CoV-2 infection in farm cats and dogs. In addition, minkto-cat transmission parameters were estimated.

\section{2 | MATERIALS AND METHODS}

\subsection{Study population and data collection}

Owners of infected mink farms (NBs) were contacted by a foundation for stray/feral cats in the Netherlands and asked to participate in this study. Ten of these farms agreed to include their dogs, (feral) cats and kittens (if present) in this research. Age, sex, pregnancy and lactating stage were registered by the veterinarians or asked of the farm owner. Additional information from the 10 mink farms was collected from records of the Netherlands Food and Consumer Product Safety Authority (NVWA) and from interviews with farm owners and workers. The precise date of viral introduction was uncertain. It was possible that the virus was already present before the mink started showing clinical signs. Therefore, to estimate the period of exposure of cats and dogs to the infected mink, the date of first clinical signs of the mink as observed by the owner and the date of culling was used. Further- more, information from interviews and from the Municipal Health Services (GGD) about the presence of any COVID-19-related symptoms in humans was included in the analyses (Oude Munnink et al., 2021). All mink farmers were asked about presence of dogs and domestic or feral cats on their farm, whether the animals were able to enter the sheds, and if the animals could come in close contact with the mink or with their food, or their bedding material.

\subsection{Sampling procedures}

Feral cats were captured using cat traps with food that were placed on and around the farm premises. The following day, the captured feral cats were sedated, neutered and treated if necessary (getting rid of flees, worms and ear mites) by veterinarians in a mobile operation unit. They were all vaccinated, chipped and eartipped. Throat swabs, rectal swabs and blood samples were taken for SARS-CoV-2 testing. Domestic cats and dogs were included for sampling if the farm owner agreed. These procedures were mostly done before or around the time the mink were culled. On the 10 participating farms a total of 101 cats (69 adults and 32 kittens) and thirteen domestic dogs were included in the study. All kittens and 59 adult cats were feral, 10 were domestic cats. In total, 114 rectal swabs and 112 throat swabs were taken. Blood collection was successful in 77 of the 114 animals, because it was not attempted in most kittens and not all domestic cats could be sedated and sampled for blood.

\section{3 | Laboratory procedures}

\subsection{1 | RT-PCR and whole genome sequencing (WGS)}

Rectal swabs and throat swabs were stored at $-80^{\circ} \mathrm{C}$ without additional medium and analysed for presence of SARS-CoV-2 RNA using real time reverse-transcription PCR using the E-gene assay (Oreshkova et al., 2020). WGS was attempted if samples had a Ct value of $\leq 32$ in the PCR test. Determination of the viral sequence was done by nextgeneration sequencing and deposited in the GISAID EpiCoV Database (https://www.gisaid.org/). Sequencing was performed to find out if cat or dog sequences belonged to the same cluster as the mink living on the same farms. The collected sequences were aligned using MAFFT v7.427 and the evolutionary history was inferred by using RAxML version 8.2.12 utilizing the Maximum Likelihood method based on the General Time Reversible model with a gamma-distributed variation of rates and 50 bootstrap replicates.

\subsubsection{ELISA and virus neutralization (VN) assay}

Serology was performed as previously described by Zhao et al. (2021). If the ELISA was found positive, the positive test was validated by a virus neutralization assay (VN) and performed as previously described (Zhao et al., 2021). A titer of $\geq 16$ was considered positive. 
TAB LE 1 Descriptive characteristics of the studied dogs, cats and kittens present on 10 infected mink farms

\begin{tabular}{|c|c|c|c|}
\hline & Cat & Kitten & Dog \\
\hline N & 69 & 32 & 13 \\
\hline Age, mean (min-max), years & $1.62(1-12)$ & $0(0-0)$ & $5.85(1-13)$ \\
\hline Domestic, \% & 13.0 & 6.2 & 100 \\
\hline Pregnant, \% & 11.8 & 0 & 0 \\
\hline PCR rectal swab+, $n(\%)$ & $0(0)$ & $0(0)$ & $0(0)$ \\
\hline PCR throat swab+ & $3(4.4)$ & $0(0)$ & $1(7.7)$ \\
\hline Ct-value PCR throat swab, mean (min-max) & $34(32-37)$ & n.a. & 33 \\
\hline Blood sample taken, $n$ & 62 & 3 & 13 \\
\hline ELISA+, PCR-, $n$ & 9 & 0 & 1 \\
\hline ELISA-, PCR,$+ n$ & 1 & 0 & 0 \\
\hline SARS-CoV-2 positive, $n(\%)$ & $12(19.4)$ & $0(0)$ & $2(15.4)$ \\
\hline
\end{tabular}

n.a. : not applicable.

\subsection{Data analysis}

Statistical analysis was performed using $R$ version 3.5.2. Data and $R$ script are available (van Aart et al., 2021). Cats were diagnosed as 'SARS-CoV-2 positive' if seropositive and/or PCR-positive. Only the adult cats with both serology results and PCR test were used for risk analysis. Two-sample t-tests and Fisher Exact tests were performed on possible risk factors (e.g. age, sex) for a positive SARS-CoV-2 test in cats.

Mink-to-cat transmission was calculated using an extreme scenario, assuming all cat infections were due to transmission by mink. Mink-tocat transmission of the virus was assumed to be constant on the days between the start of exposure $\left(t_{0}\right)$, assumed to be the date of first clinical signs in mink, and the end of exposure of a cat $\left(t_{s}\right)$, either at sampling of the cats or at culling of the mink. We quantified the transmission coefficient for transmission of an infected farm to cats on that farm ( $\beta$, infections per day) by calculating the probability of escaping the infection during an outbreak based on the prevalence $(p)$ of infected cats at the end of the outbreak: $p=1-e^{-\beta\left(t_{s}-t_{0}\right)}$ using a generalized linear model with a complementary log-log link function (Velthuis et al., 2007). We tested the hypothesis that the observed number of infections on farms differed from the expected based on the overall transmission coefficient and farm dependent exposure times with a Chisquare test.

\section{3 | RESULTS}

A total of fourteen animals had evidence of SARS-CoV-2 infection: 2 dogs and 12 adult feral cats (Table 1). These animals came from 4 of the 10 participating mink farms: NB1 (7 positive cats), NB4 (4 positive cats), NB6 (1 positive cat) and NB52 (2 positive dogs). Three throat samples of feral cats ( $3 \%$ of all cats and kittens) and one dog (8\%) were PCR-positive (Ct 32-37). All rectal swabs tested PCR-negative. Antibodies were found in 11 cats (17.7\%) and 2 dogs (15.4\%). VNTtiters ranged from 64 to 2048 (with a median of 512). Ten animals had antibodies while their swabs were negative for presence of viral RNA. Three animals were both positive for viral RNA and had SARSCoV-2 neutralizing antibodies. One feral cat had a positive throat swab, but no SARS-CoV-2 antibodies were detected the time of sampling (Table 1); we did not attempt to capture and sample this cat a second time. Age, sex, pregnancy, lactation and if the animal was feral or domestic were not associated with SARS-CoV-2 infection in the sampled cats ( $p>0.10$; data not shown).

NB1 and NB4 belonged to the same owner and were among the first diagnosed farms. The estimated SARS-CoV-2 exposure period of cats or dogs was longest for NB1 and NB4 (22-41 days), while it appeared to be shorter for NB6 and NB52 (4-8 days) and farms with no infected cats or dogs (1-19 days; Table 2). The time between the first diagnosis of the mink and sampling of cats and dogs ranged from 0 to 54 days. At all 10 participating farms, humans were also diagnosed with SARSCoV-2 infection, mostly soon after diagnosis of mink (Oude Munnink et al., 2021). Mink on all farms showed clinical signs and were positive for viral RNA. The proportion of positive throat swabs in mink was larger than the proportion of positive rectal swabs (Molenaar et al., 2020). On all farms, dogs or cats were allowed to come close to the mink, their food and their bedding material.

One of the two SARS-CoV-2-positive dogs at NB52 became very sick (fever, lethargy and anorexia) on September 2. This dog tested negative for SARS-CoV-2 RNA on September 15, whereas the other 


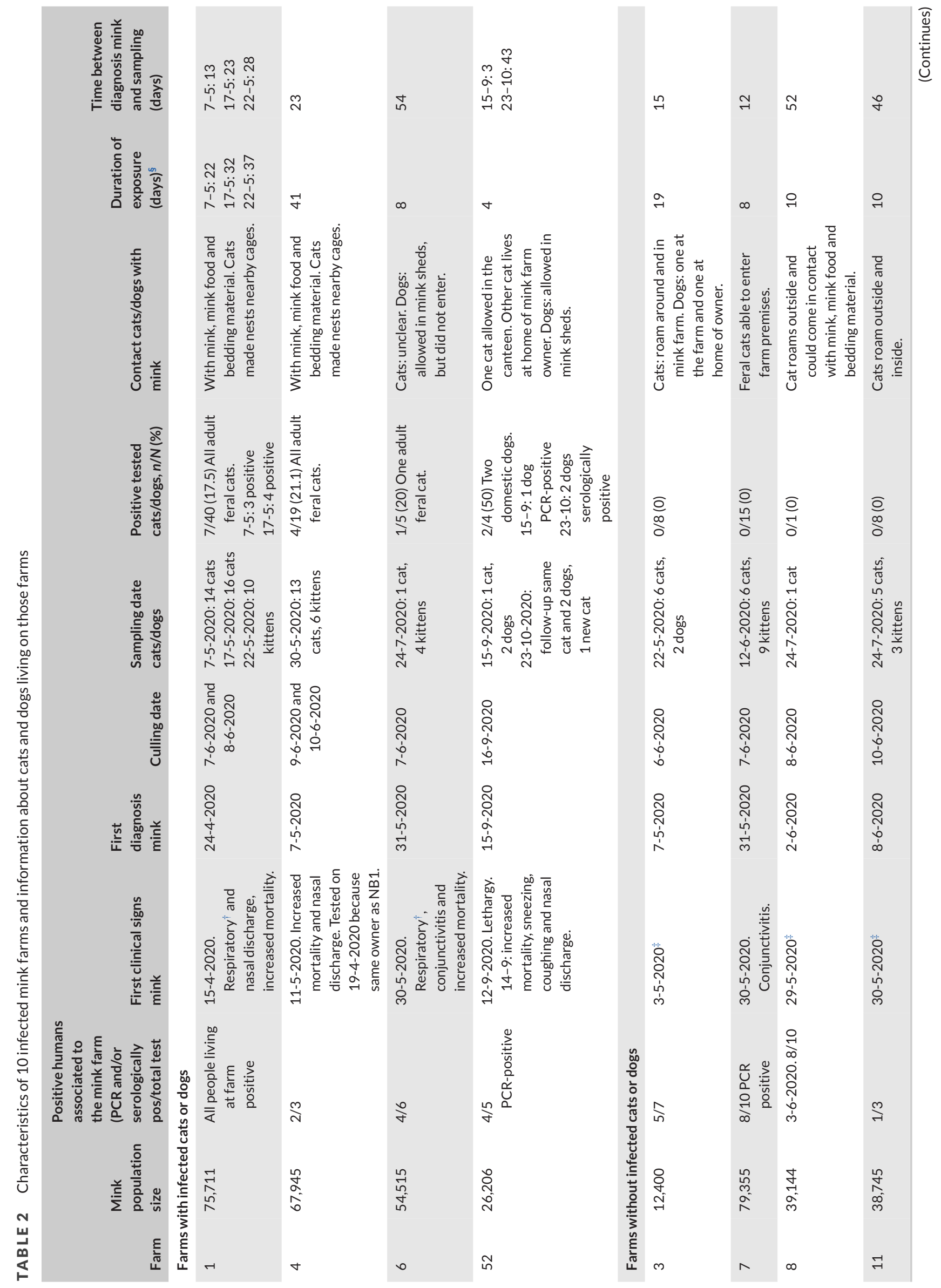




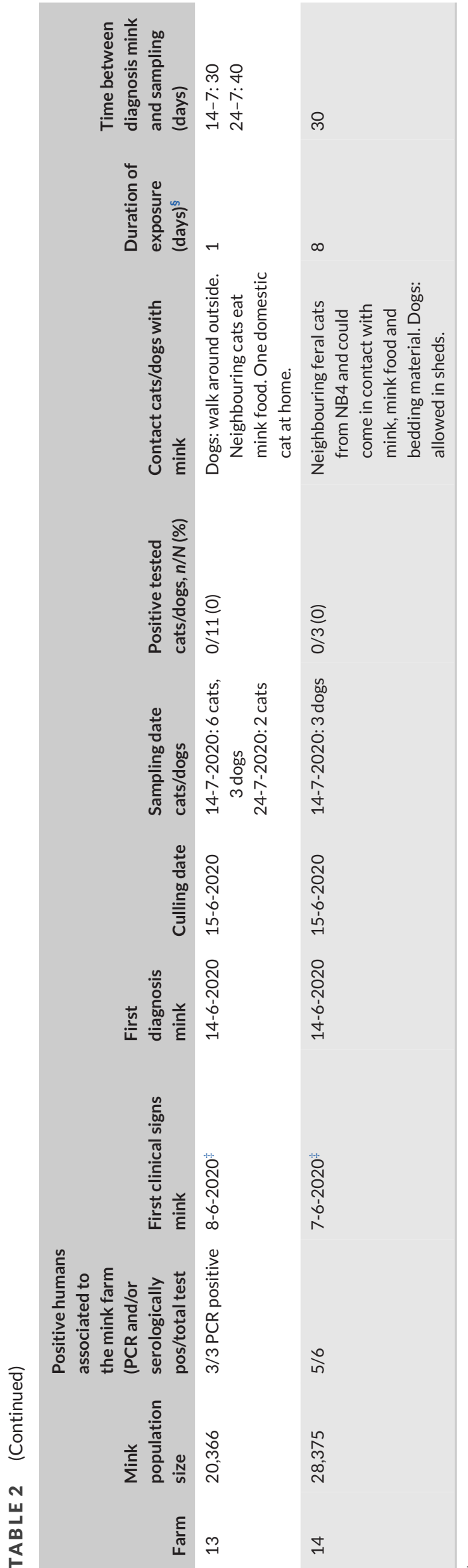

dog had no symptoms and tested PCR-positive on the same day. On September 12, the mink started showing symptoms, and also tested PCR positive on September 15. Humans working on the mink farm also tested PCR positive on September 18. On October 23 (38 days after the first sampling), a second blood sample was taken of the two dogs, and both dogs had seroconverted. Swabs of one cat at NB52 were repeated and another cat was swabbed for the first time on October 23. Both cats tested PCR negative.

One sequence was generated from a throat sample from one female feral cat captured at farm NB4. A phylogenetic tree was made to align the cat sequence with the mink sequences from the same farm, from other farms (NB1, 2, 6, 7, 8 and 10) and a selection of human sequences from the Netherlands in the period of May to August 2020 (Figure 1). The cat sequence clusters with mink sequences of NB4.

The transmission coefficient from mink-to-cat was estimated at 0.006 infections per day $(95 \% \mathrm{Cl} 0.005-0.009)$ which results in an expected prevalence in the cats of $18 \%-23 \%$ for the mean exposure time at NB1 and NB4 (31 and 41 days). The hypothesis that the transmission coefficient was the same on the 10 included farms could not be rejected $\left(\chi_{d f=9}^{2}=7.0, p=0.64\right)$.

\section{4 | DISCUSSION}

In this study, evidence of SARS-CoV-2 infection was found in 12 feral cats and 2 dogs living at mink farms where SARS-CoV-2 outbreaks among mink had occurred. A whole genome sequence generated of a cat sample clustered with mink sequences from the same farm. Prior to this study, infected cats and dogs were assumed to be infected by humans, mostly owners who were known COVID-19 patients (Patterson et al., 2020). In the present study, none of the nine domestic cats got infected, although they lived in close contact with their SARS-CoV2-positive owners. All 12 infected cats were feral cats and for these cats mink-to-cat transmission was assumed to be the most likely route of transmission. These cats roamed through the mink sheds and were likely exposed to SARS-CoV-2, either directly from mink or via airborne dust and surfaces where SARS-CoV-2 RNA has been detected (de Rooij et al., 2021). In mink, mortality cases were widespread within the farm, suggesting extensive mink-to-mink spread in the mink houses (Molenaar et al., 2020). It is highly likely that the feral cats contracted the infection from the mink or other feral cats, not from humans, as most feral cats do not seek close contact with humans. The calculated rate of mink-to-cat transmission showed that cats on infected mink farms on average had a chance of $12 \%(95 \% \mathrm{Cl} 10 \%-18 \%)$ of becoming infected by mink, considering a mean exposure time of 20 days, and assuming there was no cat-to-cat transmission. As cat-to-cat transmission could not be excluded, the estimated mink-to-cat transmission rate shows the upper limit of transmission. The six farms with no infected cats or dogs did not appear to differ from the other farms with regard to farm access, or infections in mink or humans. However, estimated exposure times were shorter compared with NB1 and NB4, the two farms with most infected cats. 


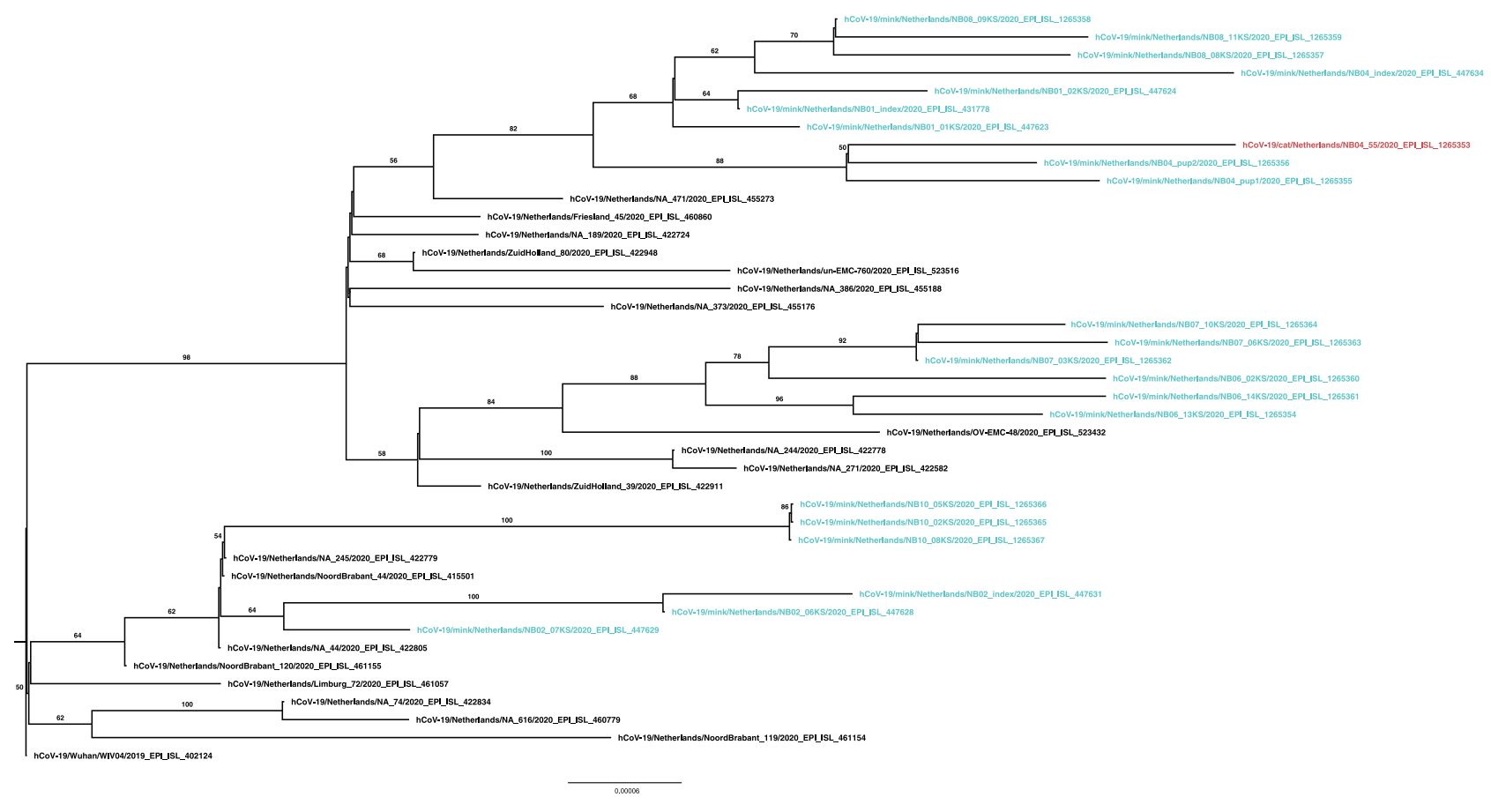

FIG URE 1 Maximum likelihood phylogenetic tree based on selected nucleotide sequence of full length SARS-CoV-2 from the GISAID EpiCoV Database (https://www.gisaid.org/) and the sequences from six mink farms. The mink sequences are blue and the cat sequence is red. The tree is rooted at Wuhan-Hu-1. Bootstrap support values above 50 are indicated at the corresponding branch

Some farms were located relatively close to each other and the possibility of farm-to-farm transmission of SARS-CoV-2 by feral cats could not be excluded, but was considered low. A previous analysis failed to show a clear geographical pattern in viral sequence clusters. For example, the mink in the farm located next to NB4 (< $500 \mathrm{~m}$ ) - a farm with several infected feral cats - had a different sequence cluster (Oude Munnink et al., 2021). However, infected cats could have transmitted the virus to other cats or susceptible wildlife species (Boklund et al., 2021).

Two dogs tested positive on a farm where four out of five humans tested positive. Human-to-dog transmission has been described before (Patterson et al., 2020; Shi et al., 2020), but we cannot exclude the possibility that the dogs - who were allowed to enter the mink sheds were infected by mink, also given the timing of clinical symptoms and diagnosed infections in dogs, mink and humans. Further research concerning the susceptibility of dogs (natural and experimental infections) is necessary to better understand the SARS-CoV-2 risk in dogs.

We did not include control farms (non-infected farms), but the observed prevalence in our study vastly exceeded the low prevalence observed in cats and dogs in the general population (Zhao et al., 2021). WGS was possible with just one sample, providing limited evidence for mink-to-cat transmission. Selection bias could have influenced study results: it was unknow how many feral cats were present at the farms. Furthermore, not all mink farms that housed cats and dogs agreed to partake in this study.

In conclusion, SARS-CoV-2-positive cats and dogs were identified on infected mink farms. The feral cats were most likely infected by mink, whereas the source of the infection in both dogs remains inconclusive. Whether this was an introduction followed by cat-to-cat transmission cannot be determined. As ongoing cat-to-cat transmission cannot be excluded, more research is needed to investigate the development of a potential reservoir in (feral) cats.

\section{ACKNOWLEDGEMENTS}

We acknowledge the submitting and originating laboratories of the sequences from GISAID's EpiCov Database on which the phylogenetic tree was based. The Municipal Health Services (GGD) are thanked for providing data on human infections at mink farms. We especially want to thank veterinarian J.W. Dijkshoorn of Pecon BV for sampling the animals at NB52. This study was commissioned and funded by the Netherlands Ministry of Agriculture, Nature and Foods.

\section{CONFLICT OF INTEREST}

Authors declare no conflict of interest.

\section{DATA AVAILABILITY STATEMENT}

All data and R script are available (van Aart et al., 2021).

\section{ETHICS STATEMENT}

According to the Dutch animal health and welfare law sampling of animals on infected premises can be carried out to examine outbreaks of notifiable diseases that may pose a risk for animal and/or public health. Sampling of the animals on infected mink farms was carried out with this respect. 


\section{ORCID}

Francisca C. Velkers (D) https://orcid.org/0000-0002-2923-3233

Egil A.J. Fischer (D) https://orcid.org/0000-0002-0599-701X

Wim H.M. van der Poel (D) https://orcid.org/0000-0002-7498-8002

Lidwien A.M. Smit (iD https://orcid.org/0000-0003-0292-0946

\section{REFERENCES}

Boklund, A., Hammer, A. S., Quaade, M. L., Rasmussen, T. B., Lohse, L., Strandbygaard, B., Jørgensen, C. S., Olesen, A. S., Hjerpe, F. B., Petersen, H. H., Jensen, T. K., Mortensen, S., Calvo-Artavia, F. F., Lefèvre, S. K., Nielsen, S. S., Halasa, T., Belsham, G. J., \& Bøtner, A. (2021). SARS-CoV-2 in Danish Mink Farms: Course of the epidemic and a descriptive analysis of the outbreaks in 2020. Animals, 11, 164. https://doi.org/10.3390/ani11010164

Decaro, N., Balboni, A., Bertolotti, L., Martino, P. A., Mazzei, M., Mira, F., \& Pagnini, U. (2021). SARS-CoV-2 infection in dogs and cats: Facts and speculations. Frontiers in Veterinary Science, 8, 619207. https://doi.org/ 10.3389/fvets.2021.619207

de Rooij, M. M. T., Hakze-van der Honing, R. W., Hulst, M. M., Harders, F., Engelsma, M., van de Hoef, W., Meliefste, K., Nieuwenweg, S., Munnink, B. B. O., van Schothorst, I., Sikkema, R. S., van der Spek, A. N., Spierenburg, M., Spithoven, J., Bouwstra, R., Molenaar, R.-J., Koopmans, M., Stegeman, A., ...Smit, L. A. M. (2021). Occupational and environmental exposure to SARS-CoV-2 in and around infected mink farms. MedRXiv. https: //doi.org/10.1101/2021.01.06.20248760.

Fenollar, F., Mediannikov, O., Maurin, M., Devaux, C., Colson, P., Levasseur, A., Fournier, P. E., \& Raoult, D. (2021). Mink, SARS-CoV-2, and the humananimal interface. Frontiers in Microbiology, 12, 663815. https://doi.org/10. 3389/fmicb.2021.663815

Halfmann, P. J., Hatta, M., Chiba, S., Maemura, T., Fan, S., Takeda, M., Kinoshita, N., Hattori, S. I., Sakai-Tagawa, Y., Iwatsuki-Horimoto, K., Imai, M., \& Kawaoka, Y. (2020). Transmission of SARS-CoV-2 in domestic cats. New England Journal of Medicine, 383, 592-594. https://doi.org/10.1056/ NEJMc2013400

Molenaar, R. J., Vreman, S., Hakze-van der Honing, R. W., Zwart, R., de Rond, J., Weesendorp, E., Smit, L. A. M., Koopmans, M., Bouwstra, R., Stegeman, A., \& van der Poel, W. H. M. (2020). Clinical and pathological findings in SARS-CoV-2 disease outbreaks in farmed mink (Neovison vison). Veterinary Pathology, 57, 653-657. https://doi.org/10.1177/ 0300985820943535

Oreshkova, N., Molenaar, R. J., Vreman, S., Harders, F., Oude Munnink, B. B., Hakze-van der Honing, R. W., Gerhards, N., Tolsma, P., Bouwstra, R., Sikkema, R. S., Tacken, M. G., de Rooij, M. M., Weesendorp, E., Engelsma, M. Y., Bruschke, C. J., Smit, L. A., Koopmans, M., van der Poel, W. H., \& Stegeman, A. (2020). SARS-CoV-2 infection in farmed minks, the Netherlands, April and May 2020. Euro Surveillance, 25, 2001005.

Oude Munnink, B. B., Sikkema, R. S., Nieuwenhuijse, D. F., Molenaar, R. J., Munger, E., Molenkamp, R., van der Spek, A., Tolsma, P., Rietveld, A., Brouwer, M., Bouwmeester-Vincken, N., Harders, F., Hakze-van der Honing, R., Wegdam-Blans, M. C. A., Bouwstra, R. J., GeurtsvanKessel, C., van der Eijk, A. A., Velkers, F. C., Smit, L. A. M., ... Koopmans, M. P. G. (2021). Transmission of SARS-CoV-2 on mink farms between humans and mink and back to humans. Science, 371, 172-177. https://doi.org/10.1126/ science.abe5901

Patterson, E. I., Elia, G., Grassi, A., Giordano, A., Desario, C., Medardo, M., Smith, S. L., Anderson, E. R., Prince, T., Patterson, G. T., Lorusso, E., Lucente, M. S., Lanave, G., Lauzi, S., Bonfanti, U., Stranieri, A., Martella, V., Solari Basano, F., Barrs, V. R., ... Decaro, N. (2020). Evidence of exposure to SARS-CoV-2 in cats and dogs from households in Italy. Nature Communications, 11, 6231. https://doi.org/10.1038/s41467-020-20097-0

Rijksoverheid (2020). Vragen over nertsen en het coronavirus. Retrieved from https://www.rijksoverheid.nl/onderwerpen/coronavirus-covid-19/ gezondheid-en-zorg/dieren/nertsen

Shi, J., Wen, Z., Zhong, G., Yang, H., Wang, C., Huang, B., Liu, R., He, X., Shuai, L., Sun, Z., Zhao, Y., Liu, P., Liang, L., Cui, P., Wang, J., Zhang, X., Guan, Y., Tan, W., Wu, G., ...Bu, Z. (2020). Susceptibility of ferrets, cats, dogs, and other domesticated animals to SARS-Coronavirus 2. Science, 368, 10161020. https://doi.org/10.1126/science.abb7015

van Aart, A. E., Velkers, F. C., Fischer, E. A. J., Broens, E. M., Egberink, H., Zhao, S., Engelsma, M., Hakze-Van der Honing, R. W., de Rooij, M. M. T., Rradstake, C., Augustijn, M., Meijer, P. A., Munnink, B. B. O., de Rond, J., Sikkema, R. S., van der Spek, A. N., Spierenburg, M., Wolters, W. J., Molenaar, R.-J., ...Smit, L. A. M. (2021). SARS-CoV-2 infection in cats and dogs in infected mink farms. Retrieved from https://github.com/EgilFischer/ mink-cat\#citation

Velthuis, A. G. J., Bouma, A., Katsma, W. E. A., Nodelijk, G., \& de Jong, M. C. M. (2007). Design and analysis of small-scale transmission experiments with animals. Epidemiology and Infection, 135(2), 202-217. https://doi.org/10. 1017/S095026880600673X

Zhao, S., Schuurman, N., Li, W., Wang, C., Smit, L. A. M., Broens, E. M., Wagenaar, J. A., van Kuppeveld, F. J. M., Bosch, B. J., \& Egberink, H. (2021). Serologic screening of severe acute respiratory syndrome coronavirus 2 infection in cats and dogs during first coronavirus disease wave, the Netherlands. Emerging Infectious Diseases, 27, 1362-1370. https://doi. org/10.3201/eid2705.204055

\section{SUPPORTING INFORMATION}

Additional supporting information may be found online in the Supporting Information section at the end of the article.

How to cite this article: Van Aart, A. E., Velkers, F. C., Fischer, E. A. J., Broens, E. M., Egberink, H., Zhao, S., Engelsma, M., Hakze-van der Honing, R. W., Harders, F., de Rooij, M. M. T., Radstake, C., Meijer, P. A., Munnink, B. B. O., de Rond, J., Sikkema, R. S., van der Spek, A. N., Spierenburg, M., Wolters, W. J., ... Smit, L. A. M. (2021). SARS-CoV-2 infection in cats and dogs in infected mink farms. Transboundary and Emerging Diseases, 1-7. https://doi.org/10.1111/tbed.14173 